\title{
Postcolonoscopy pancreatitis
}

Colonoscopy is a therapeutic and diagnostic procedure with complications commonly including intestinal perforation, splenic injury, gastrointestinal bleeding, and cardiopulmonary complications. We report the case of a 25 -year-old woman who developed acute pancreatitis following a diagnostic colonoscopy.

The patient was being followed up after a right hemicolectomy with ileocecal anastomosis for adenocarcinoma of cecum 4 months prior to presentation. She had received two cycles of chemotherapy and a course of radiotherapy 1 month previously. The provisional plan was for her to undergo surgery to complete her treatment. She therefore underwent a colonoscopy to rule out a synchronous lesion and residual disease, which was normal. The colonoscopy could not however evaluate the ascending colon because of non-distension of the bowel.

The patient developed nausea with two episodes of vomiting and epigastric pain, 4 hours after the colonoscopy. Physical examination revealed tenderness in the epigastric region but no guarding or rigidity was noted. An abdominal ultrasound performed next day was unre- markable. Laboratory test results showed markedly elevated levels of serum amylase at 1500IU/L (normal 40-140IU/L) and serum lipase at $512 \mathrm{IU} / \mathrm{L}$ (normal 0 $160 \mathrm{IU} / \mathrm{L})$ [1] confirming a diagnosis of acute pancreatitis. Other results are shown in Table 1. No computed tomography (CT) scan was performed and the patient was managed conservatively.

The common complications of colonoscopy include intestinal perforation, splenic injury [2], and, less commonly, sepsis and splenic trauma [3], among others. The most frequent causes of pancreatitis are gallstones and high alcohol intake [4]. Less frequent causes include metabolic derangements such as hypercalcemia and hypertriglyceridemia, trauma, and medications [3].

A review of the literature revealed three reported cases of colonoscopy-induced pancreatitis $[3,5,6]$. Two of the cases $[5$, 6] were technically challenging procedures: the colonoscope had been advanced beyond the splenic flexure, with excessive maneuvering and air insufflation, which was also done in our case. The third case [3] was an uncomplicated procedure. Another case of pancreatitis was reported after combined upper and lower gastrointestinal endoscopies, which were uncomplicated procedures [7].

We agree with the authors of the other case reports [3, 5-7], who propose that mechanical injury to the body and tail of pancreas by the colonoscope is the likely root cause. Further, we propose that excessive bowel distension due to gas insufflation may have an independent, if minor, role in causing colonoscopy-related pancreatitis.

\section{Endoscopy_UCTN_Code_CPL_1AJ_2AB}

Competing interests: None

\section{Skand Shekhar ${ }^{1}$, Seema Singh², Sanjay Gupta}

${ }^{1}$ University College of Medical Sciences, Delhi, India

${ }^{2}$ Department of Surgery, University College of Medical Sciences, Delhi, India

\section{References}

1 Dugdale DC. Lipase test. Medline Plus. Available at: http://www.nlm.nih.gov/medlineplus/ency/article/003465.htm. Accessed: 20 December 2013

2 Cooper GS, Kou TD, Rex DK. Complications following colonoscopy with anesthesia assistance: a population-based analysis. JAMA Intern Med 2013; 173: 551 - 556

\begin{tabular}{|c|c|c|}
\hline Test & Result & Normal range \\
\hline Hemoglobin, g/dL & 17.2 & $12-17$ \\
\hline Total leukocyte count, cells $/ \mathrm{mm}^{3}$ & 8600 & $3900-10700$ \\
\hline Platelets, cells $/ \mathrm{mm}^{3}$ & 130000 & $150000-350000$ \\
\hline Serum calcium $\left(\mathrm{Ca}^{2+}\right), \mathrm{mg} / \mathrm{dL}$ & 8.4 & $8.4-10.5$ \\
\hline Serum sodium $\left(\mathrm{Na}^{+}\right), \mathrm{mmol} / \mathrm{L}$ & 136.0 & $135.0-145.0$ \\
\hline Serum potassium $\left(\mathrm{K}^{+}\right) \mathrm{mmol} / \mathrm{L}$ & 4.0 & $3.5-5.0$ \\
\hline Blood urea, mg/dL & 23 & $15-40$ \\
\hline Serum creatinine, $\mathrm{mg} / \mathrm{dL}$ & 0.8 & $0.7-1.3$ \\
\hline Blood glucose, mg/dL & 93 & $70-110$ \\
\hline Serum bilirubin, $\mathrm{mg} / \mathrm{dL}$ & 0.7 & $0.4-1.5$ \\
\hline Serum alkaline phosphatase, IU/L & 70 & $36-92$ \\
\hline Aspartate transaminase (AST), IU/L & 31 & $0-55$ \\
\hline Alanine transaminase (ALT), IU/L & 23 & $0-48$ \\
\hline Total protein, g/dL & 4.4 & $6.0-8.0$ \\
\hline Albumin, g/dL & 2.0 & $3.1-4.3$ \\
\hline Prothrombin time, seconds & 12.5 & $11-13$ \\
\hline Activated partial thromboplastin time (APTT), seconds & 28.1 & $25-35$ \\
\hline Serum cholesterol, mg/dL & 99 & $<200$ \\
\hline $\mathrm{HDL}, \mathrm{mg} / \mathrm{dL}$ & 28 & $>40$ \\
\hline VLDL, mg/dL & 11 & $2-30$ \\
\hline $\mathrm{LDL}, \mathrm{mg} / \mathrm{dL}$ & 60 & $<130$ \\
\hline Triglycerides, mg/dL & 54 & $<150$ \\
\hline
\end{tabular}

Table 1 Results of blood tests taken after the patient developed vomiting and epigastric pain 4 hours after undergoing colonoscopy. 
3 Khashram M, Frizelle FA. Colonoscopy - a rare cause of pancreatitis. NZ Med J 2011; 124: 74-76

4 Frossard JL, Steer ML, Pastor CM. Acute pancreatitis. Lancet 2008; 371: $143-152$

5 Thomas AW, Mitre RJ. Acute pancreatitis as a complication of colonoscopy. Clin Gastroenterol 1994; 19: 177 - 188

6 Ko HH, Jamieson T, Bressler B. Acute pancreatitis and ileus postcolonoscopy. Can J Gastroenterol 2009; 23: $551-553$

7 Nevins AB, Keeffe EB. Acute pancreatitis after gastrointestinal endoscopy. J Clin Gastroenterol 2002; 34: 94-95
Bibliography

DOI http://dx.doi.org/

10.1055/s-0034-1377379

Endoscopy 2014; 46: E515-E516

(C) Georg Thieme Verlag KG

Stuttgart · New York

ISSN 0013-726X
Corresponding author

\section{Skand Shekhar}

Medical Education Unit 2nd Floor, Library Block UCMS and GTB Hospital Dilshad Garden New Delhi-110095 India

Fax: +91-11-26493777

skand.shekhar@gmail.com 